Интернет-журнал «Транспортные сооружения» https://t-s.today

Russian journal of transport engineering

2019, №4, Том 6 / 2019, No 4, Vol 6 https://t-s.today/issue-4-2019.html

URL статьи: https://t-s.today/PDF/05SATS419.pdf

DOI: 10.15862/05SATS419 (http://dx.doi.org/10.15862/05SATS419)

Ссылка для цитирования этой статьи:

Александровский М.В. Использование обобщенного метода конечных разностей для расчета балок из нелинейно-упругого материала // Интернет-журнал «Транспортные сооружения», 2019 №4, https://t-s.today/PDF/05SATS419.pdf (доступ свободный). Загл. с экрана. Яз. рус., англ. DOI: $10.15862 / 05$ SATS419

For citation:

Aleksandrovskiy M.V. (2019). Use of generalized finite difference method for calculation of beams from nonlinear elastic material. Russian journal of transport engineering, [online] 4(6). Available at: https://t-s.today/PDF/05SATS419.pdf (in Russian). DOI: 10.15862/05SATS419

\title{
УДК 624.072
}

Александровский Максим Вячеславович ФГБОУ ВО «Национальный исследовательский Московский государственный строительный университет» Москва, Россия

Доцент кафедры «Строительной и теоретической механики» Кандидат технических наук E-mail: AleksandrovskiyMV@mgsu.ru

\section{Использование обобщенного метода конечных разностей для расчета балок из нелинейно-упругого материала}

Аннотация. Внедрение в практику строительства конструкций из высокопрочных сталей и других материалов, имеющих нелинейную диаграмму деформирования, вызвало активное развитие нелинейной теории расчета конструкций. Замена закона Гука нелинейными зависимостями между напряжениями и деформациями приводит к так называемой физической нелинейности. Для расчета таких конструкций экспериментально полученные зависимости между напряжениями и деформациями описываются с помощью аналитических выражений. Различными исследователями был предложен ряд вариантов таких аппроксимаций. В представленной работе рассматривается расчет балок симметричного сечения, выполненных из нелинейно-упругого материала, для которого зависимость между напряжениями и деформациями описывается кубической параболой. Такая аппроксимация обеспечивает симметричность диаграммы $\sigma-\varepsilon$ относительно растяжения - сжатия, а также дает хорошее совпадение с экспериментальной кривой. Использование для решения поставленной задачи обобщенного метода конечных разностей позволяет свести систему нелинейных дифференциальных уравнений к системе алгебраических уравнений, для решения которой используется метод последовательных приближений. Исследования показали, что предложенный метод расчета дает возможность получить достаточно точное решение при небольшом числе элементов. Кроме того, представленный алгоритм расчета удобен для программирования и численного счета. В качестве примера систем, допускающих расчет с использованием рассмотренного алгоритма, могут выступать балочные элементы строительных конструкций. Приведен расчет балки из нелинейно-упругого материала на действие сосредоточенной силы. 
Ключевые слова: алгоритм расчёта; нелинейно-упругий материал; аппроксимация кубической параболой; обобщенный метода конечных разностей; система нелинейных дифференциальных уравнений; система алгебраических уравнений

\section{Введение}

При изложении классических методов расчета, в подавляющем числе случаев предполагается, что материал конструкций работает в упругой стадии, то есть между напряжениями и деформациями соблюдается закон Гука.

В настоящее время, в практику строительства активно внедряются конструкции из высокопрочных сталей и других материалов, имеющих нелинейную диаграмму деформирования. Замена закона Гука нелинейными зависимостями между напряжениями и деформациями приводит к так называемой физической нелинейности [1]. Физически нелинейные задачи и относящиеся к ним вопросы рассмотрены, например в работах [2-9].

Для расчета таких конструкций экспериментально полученные зависимости между напряжениями и деформациями описываются с помощью аналитических выражений. Различными исследователями был предложен ряд вариантов аппроксимации диаграммы деформирования $\sigma-\varepsilon$.

\section{Методология}

В представленной работе рассматривается расчет балки симметричного сечения, выполненной из нелинейно-упругого материала, для которого зависимости между напряжениями и деформациями описываются кубической параболой [8]. Такая аппроксимация обеспечивает симметричность диаграммы $\sigma-\varepsilon$ относительно растяжения - сжатия, а при деформациях, стремящихся к нулю, она переходит в закон Гука.

Для решения нелинейных дифференциальных уравнений, описывающих напряженно деформированное состояние балки, используется обобщенный метод конечных разностей, идеи которого изложены в [10]. Данный метод позволяет учитывать разрывы функций и их производных без специального сгущения расчетных точек и использования законтурных точек [11]. Решение полученной в окончательном виде системы нелинейных алгебраических уравнений проводится методом последовательных приближений. Представленный алгоритм расчета удобен для программирования и численного счета.

\section{Основная часть}

Кубическую параболу, описывающую диаграмму $\sigma$ - $\varepsilon$, можно представить в следующем виде

$$
\sigma=A_{1} \varepsilon-A_{3} \varepsilon^{3}
$$

Здесь $\sigma$ и $\varepsilon$ соответственно - продольные напряжения и деформация, в поперечном сечении балки. Коэффициенты $A_{1}$ и $A_{3}$, имеющие размерность напряжения, могут быть определены различными способами в зависимости от конкретной экспериментальной кривой и поставленной задачи. Некоторые варианты для их определения представлены в работах [1] и [8].

Если длина балки значительно превышает высоту поперечного сечения и изгибающие моменты по ее длине меняются достаточно медленно, то независимо от физико-механических свойств материала, справедлива гипотеза плоских сечений. Согласно этой гипотезе, продольная 
деформация волокна, отстоящего на расстоянии $z$ от нейтрального слоя, определяется формулой

$$
\varepsilon_{z}=z / \rho,
$$

где $\rho$ - радиус кривизны изогнутой оси балки.

Подставляя (2) в (1), представим выражение для напряжения в следующем виде

$$
\sigma_{z}=A_{1} \frac{z}{\rho}-A_{3} \frac{z^{3}}{\rho^{3}} .
$$

На рисунке 1 показано прямоугольное сечение и соответствующая (3) эпюра нормальных напряжений.

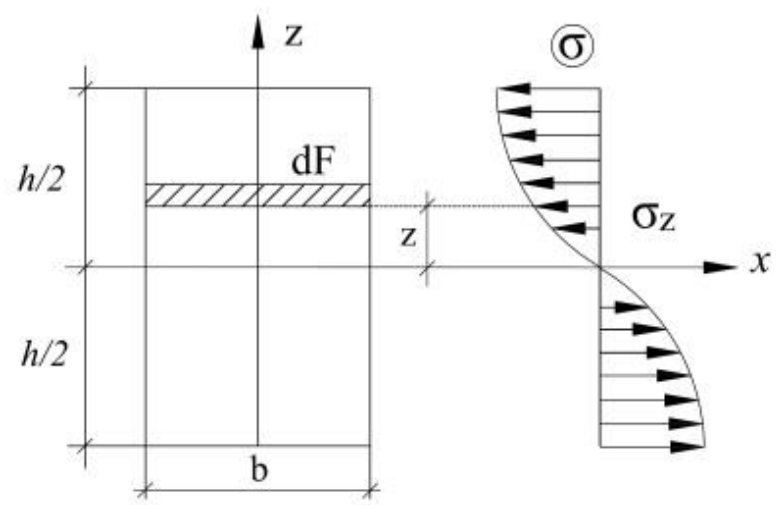

Рисунок 1. Эпюра нормальных напряжений для прямоугольного сечения

Изгибающий момент в сечении с учетом (3) определяется следующим образом:

$$
M=\int_{F} \sigma_{z} z d F=\frac{A_{1}}{\rho} \int_{F} Z^{2} d F-\frac{A_{3}}{\rho^{3}} \int_{F} Z^{4} d F,
$$

где $F$ - площадь поперечного сечения.

Введем обозначения:

$$
J_{2}=\int_{F} z^{2} d F, J_{4}=\int_{F} z^{4} d F .
$$

Здесь $J_{2}$ и $J_{4}$ - моменты инерции 2-го 4 го порядка, соответственно.

Тогда выражение (4) примет вид.

$$
M=A_{1} \frac{J_{2}}{\rho}-A_{3} \frac{J_{4}}{\rho^{3}} .
$$

Для прямоугольного сечения выражения для $J_{2}$ и $J_{4}$ будут иметь вид:

$$
J_{2}=\frac{b h^{3}}{12}, J_{4}=\frac{b h^{5}}{80} \text {. }
$$

Для геометрически линейной задачи воспользуемся известным выражением для кривизны балки

$$
\frac{1}{\rho}=-\frac{d^{2} y}{d x^{2}}
$$

где $y$ - вертикальное перемещение.

Тогда уравнение (6), связывающее изгибающий момент и кривизну, примет вид

$$
M(x)=-A_{1} J_{2} \frac{d^{2} y}{d x^{2}}+A_{3} J_{4}\left(\frac{d^{2} y}{d x^{2}}\right)^{3} .
$$


Введем новую переменную

$$
v=A_{1} J_{2} y \text { или } y=v / J_{2} y \text {. }
$$

В этом случае уравнение (9) можно представить в виде

$$
\frac{d^{2} v}{d x^{2}}=-M+\alpha\left(\frac{d^{2} v}{d x^{2}}\right)^{3}
$$

где

$$
\alpha=\frac{A_{3} J_{4}}{\left(A_{1} J_{2}\right)^{3}} .
$$

Как известно, уравнение равновесия элемента балки имеет вид

$$
\frac{d^{2} M(x)}{d x^{2}}=-q(x)
$$

где $q(x)$ - поперечная нагрузка.

Для совместного решения дифференциальных уравнений (11) и (13) используется обобщенный метод конечных разностей [10]. На рисунке 2 изображен пролет балки, который разбивается на $n$ элементов длиной - $\tau$, в пределах которых, распределенная нагрузка непрерывна, а на их границах приложены вертикальные сосредоточенные силы $\Phi_{i}$ и моменты $m_{i}$. Номер каждого элемента определяется номером правого узла.

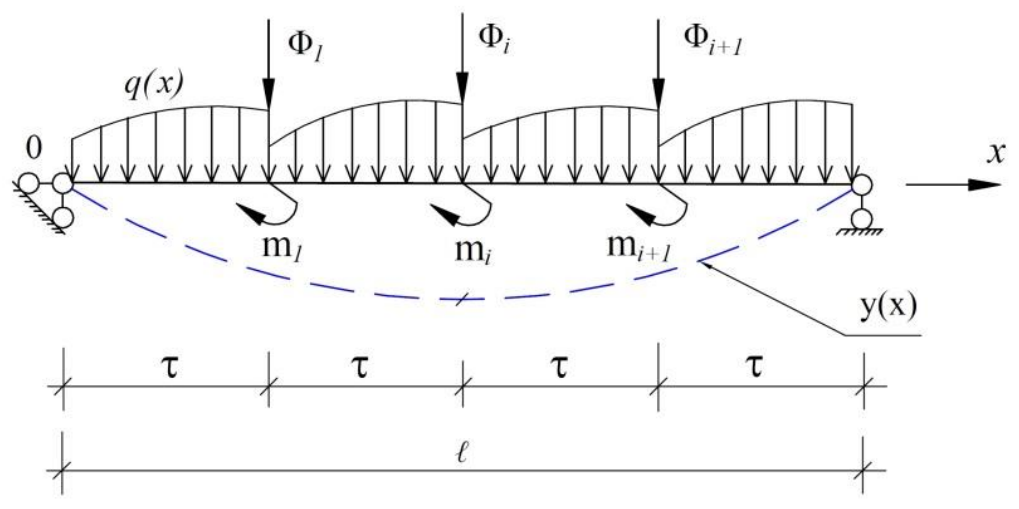

Рисунок 2. Расчетная схема балки

Введем обозначения:

$$
\begin{gathered}
\frac{d v}{d x}=\varphi ; \frac{d^{2} v}{d x^{2}}=\frac{d \varphi}{d x}=t \\
\frac{d M}{d x}=\theta ; \frac{d^{2} M}{d x^{2}}=\frac{d Q}{d x}=f,
\end{gathered}
$$

где $Q$ - поперечная сила.

Тогда уравнения (11) и (13) принимают вид:

$$
t=-M+\alpha t^{3} ; f=-q .
$$

Для решения первого уравнения (16), нелинейного относительно $t$, воспользуемся методом последовательных приближений. В этом случае $k$-ое приближение можно представить в виде

$$
t^{(k)}=-M+\alpha\left(t^{(k-1)}\right)^{3} .
$$

В качестве первого приближения берется линейное решение $t^{(1)}=M$. Во втором и третьем приближении получим, соответственно: 


$$
t^{(2)}=-\left(M+\alpha M^{3}\right) ; t^{(3)}=-M-\alpha\left(M+\alpha M^{3}\right)^{3} .
$$

Представим (17) в следующем виде

$$
t^{(k)}=-R^{(k)},
$$

где

$$
R^{(1)}=M ; R^{(2)}=M+\alpha M^{3}, R^{(3)}=M+\alpha\left(M+\alpha M^{3}\right)^{3}, \ldots .
$$

Следует отметить, что в зависимости от конкретной задачи можно ограничиться вторым или третьим приближением. Тогда систему (16) можно представить в следующем виде:

$$
t^{(k)}=-R^{(k)} ; f=-q .
$$

Получим решение второго уравнения системы (21). Следует отметить, что функции $M$, $Q, q$ непрерывны в пределах элемента, а на его границах могут иметь скачки. Рассмотрим два соседних элемента представленных на рисунке 3.

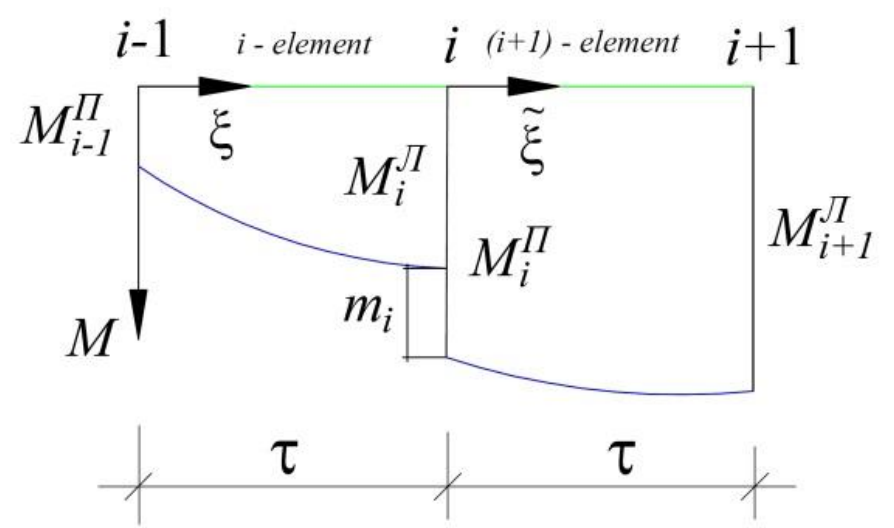

Рисунок 3. Расчетная схема двух сопряженных элементов

В пределах каждого элемента функция $M$ аппроксимируется квадратной параболой

$$
M(\xi)=a_{0}+a_{1} \xi+a_{2} \xi^{2},
$$

а ее производные имеют вид:

$$
Q=M^{\prime}=a_{1}+2 a_{2}, f=M^{\prime \prime}=2 a_{2} .
$$

Коэффициенты $a_{i}$ выражаются через значения функции $M$ на границах элементов и через производную $Q$ в узле $i$. Для элемента $i$ используется $Q_{i}^{Л}-$ производная слева, а для элемента $(i+1)$ - производная справа $-Q_{i}^{\Pi}$. Таким образом для элемента $-i$ получим следующие зависимости, определяющие соответствующие коэффициенты:

$$
a_{0}=M_{i-1}^{\Pi}, a_{1}=-Q_{i}^{\pi}+\frac{2}{\tau}\left(M_{i}^{\pi}-M_{i-1}^{\Pi}\right), a_{2}=\frac{Q_{i}^{\Pi}}{\tau}-\frac{1}{\tau^{2}}\left(M_{i}^{\Pi}-M_{i-1}^{\Pi}\right) .
$$

для элемента $-i+1$ :

$$
\widetilde{a_{0}}=M_{i-1}^{\Pi}, \widetilde{a_{1}}=Q_{i}^{\Pi}, \widetilde{a_{2}}=-\frac{Q_{i}^{\Pi}}{\tau}-\frac{1}{\tau^{2}}\left(M_{i}^{\Pi}-M_{i+1}^{\Pi}\right) .
$$

Вторая производная $f$ для каждого элемента будет определяться:

$$
\begin{aligned}
f_{i} & =\frac{2}{\tau} Q_{i}^{J}-\frac{2}{\tau^{2}}\left(M_{i}^{Л}-M_{i-1}^{\Pi}\right), \\
\mathrm{f}_{\mathrm{i}+1} & =-\frac{2}{\tau} \mathrm{Q}_{\mathrm{i}}^{\Pi}-\frac{2}{\tau^{2}}\left(\mathrm{M}_{\mathrm{i}}^{\Pi}-\mathrm{M}_{\mathrm{i}+1}^{\pi}\right) .
\end{aligned}
$$


Удовлетворим второму уравнению системы (21) слева и справа в узле $i$, то есть для элементов $i$ и $i+1$. Подставив (25) в (21), переходим к следующим зависимостям.

$$
\begin{gathered}
\frac{2}{\tau} Q_{i}^{\Pi}-\frac{2}{\tau^{2}}\left(M_{i}^{\pi}-M_{i-1}^{\Pi}\right)=-q_{i}^{\pi}, \\
-\frac{2}{\tau} Q_{i}^{\Pi}-\frac{2}{\tau^{2}}\left(M_{i}^{\Pi}-M_{i+1}^{\pi}\right)=-q_{i}^{\Pi} .
\end{gathered}
$$

Сложив эти уравнения и введя обозначения:

$$
M_{i}^{\Pi}=M_{i}^{Л}+m_{i}, Q_{i}^{\Pi}=Q_{i}^{Л}-\Phi_{i},
$$

получим разрешающее уравнение для изгибающих моментов

$$
-M_{i-1}^{\Pi}+2 M_{i}^{Л}-M_{i+1}^{\Pi}=-m_{i}+\tau \Phi_{i}+\frac{\tau^{2}}{2}\left(q_{i}^{\pi}+q_{i}^{\Pi}\right) .
$$

Здесь $m_{i}$ и $\Phi_{i}-$ сосредоточенный момент и сила в узле $i$, соответственно.

Решение первого уравнения системы (21) можно получить из (28) с заменой $M$ на $v, Q$ на $\varphi, q$ на $R$ и $\Phi_{i}$ на $\Delta \varphi_{i}=\varphi_{i}^{Л}-\varphi_{i}^{\Pi}$ (при наличии в балке промежуточных шарниров). С учетом непрерывности функции $v$ в узловых точках получим следующее уравнение для определения перемещений:

$$
-v_{i-1}+2 v_{i}-v_{i+1}=\tau \Delta \varphi_{i}+\frac{\tau^{2}}{2}\left(R_{i}^{Л}+R_{i}^{\Pi}\right)
$$

где $R_{i}$ определяется по формуле (20) для узла $i$. Для удобства записи здесь отброшен верхний индекс $(k)$, обозначающий номер итерации.

Уравнения (28) и (29) записываются для всех внутренних точек, что приводит к системе $2(n-1)$ уравнений, при этом, к основным уравнениям нужно добавить граничные условия. Из уравнений (26) получим:

$$
Q_{i}^{Л}=\frac{1}{\tau}\left(M_{i}^{\pi}-M_{i-1}^{\Pi}\right)-\frac{\tau}{2} q_{i}^{\pi}, Q_{i}^{\Pi}=-\frac{1}{\tau}\left(M_{i}^{\Pi}-M_{i+1}^{\pi}\right)+\frac{\tau}{2} q_{i}^{\Pi} .
$$

При замене $Q$ на $\varphi, M$ на $v$ и $q$ на $R$ приходим к следующим выражениям:

$$
\varphi_{i}^{\Pi}=\frac{1}{\tau}\left(v_{i}-v_{i-1}\right)-\frac{\tau}{2} R_{i}^{\Pi}, \varphi_{i}^{\Pi}=-\frac{1}{\tau}\left(v_{i}-v_{i+1}\right)+\frac{\tau}{2} R_{i}^{\Pi} .
$$

При шарнирном опирании на левой и правой опорах $v_{0}=M_{0}=0$ и $v_{n}=M_{n}=0$, что дает возможность сначала решить систему (28), определив значения $M_{i}$, а затем из решения системы (29) определить перемещения $v_{i}$.

На левом свободном крае $M_{0}^{\Pi}=0$ и $Q_{0}^{\Pi}=0$, а на правом $-M_{n}^{Л}=0$ и $Q_{n}^{Л}=0$. В соответствии с (30) при $i=0$ и $i=n$ приходим к следующим соотношениям:

$$
M_{1}^{Л}=-\frac{\tau^{2}}{2} q_{0}^{\Pi} ; M_{n-1}^{\Pi}=-\frac{\tau^{2}}{2} q_{n}^{Л} .
$$

Следует отметить, что в консольной балке для определения $M_{i}$ также достаточно уравнений (28) и одного из выражений (32).

При жестком защемлении на левом краю $-v_{0}=0$ и $\varphi_{0}^{\Pi}=0$, а на правом $-v_{n}=0$ и $\varphi_{n}^{Л}=0$. Тогда из (31) получим следующие соотношения:

$$
v_{1}=-\frac{\tau}{2} R_{0}^{\Pi} ; v_{n-1}=-\frac{\tau}{2} R_{n}^{J} .
$$

Следует отметить, что в статически неопределимых балках, например, в однопролетных балках с одним или двумя защемленными краями, для определения изгибающих моментов $M_{i}$ и перемещений $v_{i}$ необходимо совместное решение систем (28) и (29) с учетом 
дополнительных уравнений (33). Кроме того, в соответствии с (20) задача осложняется нелинейной зависимостью между $R_{i}$ и $M_{i}$. Для решения полученной системы нелинейных уравнений используется метод итераций, в котором в первом приближении считается, что $R_{i}=M_{i}$.

При отсутствии в балке промежуточных шарниров и сосредоточенных моментов, а также при $q_{i}=$ const в пределах $i$-го элемента, уравнения (28), (29), (32) и (33) принимают вид:

$$
\begin{gathered}
-M_{i-1}+2 M_{i}-M_{i+1}=\tau \Phi_{i}+\frac{\tau^{2}}{2}\left(q_{i}+q_{i+1}\right), \\
-v_{i-1}+2 v_{i}-v_{i+1}=\tau^{2} R_{i}^{(k)}, \\
M_{1}=-\frac{\tau}{2} q_{1} ; M_{n-1}=-\frac{\tau}{2} q_{n}, \\
v_{1}=-\frac{\tau}{2} R_{0}^{(k)} ; v_{n-1}=-\frac{\tau}{2} R_{n}^{(k)} .
\end{gathered}
$$

\section{Выводы}

В качестве практической реализации рассмотренного алгоритма, приведем пример расчета шарнирно опертой балки прямоугольного сечения, загруженной сосредоточенной силой $\Phi=96$ кН, приложеной в середине пролета (рис. 4). Зададимся значением величины пролета балки $\ell=8 \mathrm{M}$. Размеры поперечного сечения: $b=0,3 \mathrm{~m}$ и $h=0,4 \mathrm{~m}$. Экспериментальная зависимость $\sigma-\varepsilon$ для материала, из которого выполнена балка, представлена в [8]. Для рассматриваемого случая предел прочности материала определен величиной $-\sigma_{\text {пр }}=2 \cdot 10^{4} \mathrm{\kappa H} / \mathrm{m}^{2}$, а соответствующая ему деформация $-\varepsilon_{\text {пр }}=2 \cdot 10^{-3}$.

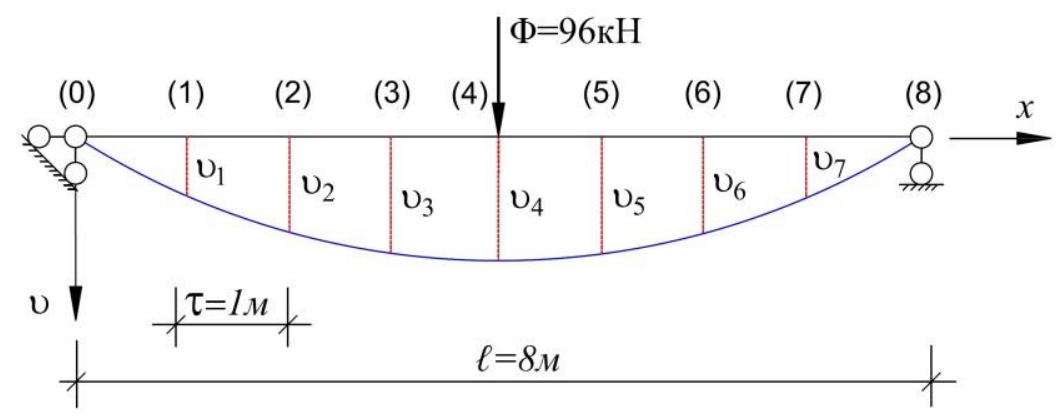

\section{Рисунок 4. Расчетная схема балки, загруженной сосредоточенной силой}

Коэффициенты $A_{1}$ и $A_{3}$ в выражении (1) в данном случае определяются из условия

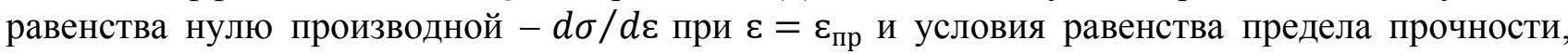
определяемого по экспериментальной диаграмме и по аппроксимирующей ее кривой при $\varepsilon=\varepsilon_{\text {пр }}$, и вычисляются по формулам:

$$
A_{1}=\frac{3}{2} \frac{\sigma_{\text {пр }}}{\varepsilon_{\text {пр }}} ; A_{3}=\frac{3}{2} \frac{\sigma_{\text {пр }}}{2 \varepsilon_{\text {пр }}^{3}} .
$$

При предложенном способе определения коэффициентов $A_{1}$ и $A_{3}$ получается очень хорошее совпадение экспериментальной и аппроксимирующей диаграммы $\sigma-\varepsilon$.

Используемые в расчете величины, определяемые формулами (7), (38), (10) и (12) примут соответствующие численные значения:

$$
J_{2}=1,6 \cdot 10^{-3} \mathrm{M}^{4} ; J_{4}=3,84 \cdot 10^{-5} \mathrm{~m}^{6} ; A_{1}=1,5 \cdot 10^{7} \kappa \mathrm{H} / \mathrm{m}^{2} ; A_{3}=1,25 \cdot 10^{12} \mathrm{\kappa H} / \mathrm{м}^{2} ;
$$
$A_{1} J_{2}=2,4 \cdot 10^{4} \mathrm{\kappa Hм}^{2} ; \alpha=3,4722 \cdot 10^{-6}(\mathrm{\kappa Hм})^{-2}$. 
Балка разбивается на 8 элементов длиной $\tau=1 \mathrm{M}$. Граничные условия запишутся в следующем виде: $M_{0}=M_{8}=0, v_{0}=v_{8}=0$. Как уже отмечалось ранее, в статически определимых системах возможно независимое определение изгибающих моментов и перемещений.

Уравнения (34), составленные с учетом симметрии балки (при $M_{3}=M_{5}$ ) для узлов $1-4$, приводит к следующей системе уравнений для определения $M_{i}$

$$
\left\{\begin{array}{c}
i=1,2 M_{1}-M_{2}=0 \\
i=2,-M_{1}+2 M_{2}-M_{3}=0 \\
i=3,-M_{2}+2 M_{3}-M_{4}=0 \\
i=4,-M_{3}+2 M_{4}-M_{3}=\Phi \tau .
\end{array}\right.
$$

Из решения системы (39) получаем:

$$
M_{1}=\frac{\Phi \tau}{2}=48, M_{2}=\Phi \tau=96, M_{3}=\frac{3}{2} \Phi \tau=144, M_{4}=2 \Phi \tau=192,
$$

что совпадает с точным решением.

Уравнения для определения $v_{0}$ с учетом тог, что $v_{5}=v_{3}$, принимают вид:

$$
\left\{\begin{array}{c}
i=1,2 v_{1}-v_{2}=\tau^{2} R_{1}^{(k)}, \\
i=2,-v_{1}+2 v_{2}-v_{3}=\tau^{2} R_{2}^{(k)}, \\
i=3,-v_{2}+2 v_{3}-v_{4}=\tau^{2} R_{3}^{(k)}, \\
i=4,-v_{3}+2 v_{4}-v_{3}=\tau^{2} R_{4}^{(k)} .
\end{array}\right.
$$

Решение этой системы можно представить в следующем виде:

$$
\left\{\begin{array}{c}
v_{1}=\tau^{2}\left(R_{1}^{(k)}+R_{2}^{(k)}+R_{3}^{(k)}+\frac{1}{2} R_{4}^{(k)}\right), \\
v_{2}=\tau^{2}\left(R_{1}^{(k)}+2 R_{2}^{(k)}+2 R_{3}^{(k)}+R_{4}^{(k)}\right), \\
v_{3}=\tau^{2}\left(R_{1}^{(k)}+2 R_{2}^{(k)}+3 R_{3}^{(k)}+\frac{3}{2} R_{4}^{(k)}\right), \\
v_{4}=\tau^{2}\left(R_{1}^{(k)}+2 R_{2}^{(k)}+3 R_{3}^{(k)}+2 R_{4}^{(k)}\right) .
\end{array}\right.
$$

Далее рассматривается определение перемещений с помощью метода последовательных приближений. Следует отметить, что верхний индекс $(k)$ в (41) и (42) означает номер итерации.

Рассмотрим первое приближение. В этом случае в соответствии с (20) $R_{i}^{(1)}=M_{i}^{(1)}$. Формулы (42) дают возможность определить перемещения во всех во всех узловых точках. На данном этапе ограничимся определением $v_{2}^{1}$ и $v_{4}^{1}$. Тогда, в соответствии с (40) и (42) получим:

$$
\begin{aligned}
& v_{2}^{(1)}=7,5 \Phi \tau^{3}=\frac{7,5}{512} \Phi \ell^{3}=1,465 \cdot 10^{-2} \Phi \ell^{3},\left(1,465 \cdot 10^{-2} \Phi \ell^{3}\right) ; \\
& v_{4}^{(1)}=11 \Phi \tau^{3}=\frac{11}{512} \Phi \ell^{3}=2,148 \cdot 10^{-2} \Phi \ell^{3},\left(2,083 \cdot 10^{-2} \Phi \ell^{3}\right) .
\end{aligned}
$$

В скобках указаны значения, полученные при аналитическом решении линеаризованного уравнения (11). Численное значение $v_{2}$ отличается от точного решения на $2,3 \%$, а $v_{4}$ на $3,12 \%$. Увеличивая число элементов, можно получить более точный результат.

С учетом (10) получим:

$$
v_{4}^{(1)}=11 \Phi \tau^{3}=11 \cdot 96 \cdot 1^{3}=1056 ; y_{4}^{(1)}=\frac{v_{4}^{(1)}}{A_{1} J_{2}}=\frac{1056}{2,4 \cdot 10^{4}}=4,4 \cdot 10^{-2} \mathrm{M} .
$$


Во втором приближении, где $R^{(2)}$ вычисляется в соответствии с (20), а также с учетом вычисленного значения - $\alpha$, используя (40) и (42), определим численное значение величины $v_{4}^{(2)}$.

$$
v_{4}^{(2)}=11 \Phi \tau^{2}+28,25 \alpha \Phi^{3} \tau^{5}=1056+28,25 \cdot 3,4722 \cdot 10^{-6} \cdot 96^{3} \cdot 1^{5}=1056+86,78=1142,78
$$

Таким образом, нелинейная составляющая перемещения составляет $8,2 \%$ от линейной величины.

В третьем приближении, согласно описанной выше процедуре вычислений, учитывающей использование формул: (20), (40) и (42), а также вычисленного значения $-\alpha$, получим численное значение для $v_{4}^{(3)} \cdot v_{4}^{(3)}=1056+86,78+29,31=1172,01$.

Так как в третьем приближении значение $v_{4}^{(3)}$ увеличилось лишь на $1,7 \%$ по сравнению с линейным решением, то в большинстве случаев можно ограничиться третьим приближением. Это позволит упростить вычисления при незначительной потере точности.

Таким образом, в статье представлен алгоритм расчёта балок симметричного сечения, выполненных из нелинейно-упругого материала, загруженных распределенной вертикальной нагрузкой, а также сосредоточенными силами и моментами. Следует отметить, что балки могут быть как однопролетными, так и многопролетными с промежуточными шарнирами.

Использование для решения поставленной задачи обобщенного метода конечных разностей позволяет свести систему нелинейных дифференциальных уравнений к системе алгебраических уравнений, для решения которой используется метод последовательных приближений. Сравнение результатов расчета для приведенного в работе примера с аналогичным решением, полученным аналитическим методом, показало, что метод обобщенных конечных разностей дает возможность получить достаточно точное решение при небольшом числе элементов. Кроме того, представленный алгоритм расчета удобен для программирования и численного счета.

Примерами систем, для которых может быть использован представленный алгоритм расчета, могут быть балочные элементы строительных конструкций.

Представленную работу, по нашему мнению, можно рассматривать как некоторый вклад в развитие методов расчета конструкций, выполненных из нелинейно-упругого материала. 


\section{ЛИТЕРАТУРА}

1. Лукаш П.А. Основы нелинейной строительной механики. М.: Стройиздат, 1978. $208 \mathrm{c}$.

2. Бондарь Н.Г. Нелинейные автономные задачи механики упругих систем. Киев: Будівельник, 1971. 140 с.

3. Цурпал И.А. Расчет элементов конструкций из нелинейно упругих материалов. Киев: «Техніка», 1976. 176 с.

4. M.A. Crisfield, Non-linear Finite Element Analysis of Solids and Structures, vol. 1, Wiley, UK, pp. 362, 1994.

5. K. Lee, "Large deflections of cantilever beams of non-linear elastic material under a combined loading", International Journal of Non-Linear Mechanics, vol. 37, no. 3, pp. 439-443, 2002.

6. M. Brojan, T. Videnic, and F. Kosel, "Large deflections of nonlinearly elastic nonprismatic cantilever beams made from materials obeying the generalized Ludwick constitutive law", Meccanica, vol. 44, no. 6, pp. 733-739, 2009.

7. E. Solano-Carrillo, "Semi-exact solutions for large deflections of cantilever beams of non-linear elastic behaviour", International Journal of Non-Linear Mechanics, vol. 44, no. 2, pp. 253-256, 2009.

8. Петров В.В., Кривошейн И.В. Методы расчета конструкций из нелинейнодеформируемого материала. М.: Изд-во АСВ, 2009. 206 с.

9. X. Zhang and J. Yang, "Inverse problem of elastica of a variable-arc-length beam subjected to a concentrated load", Acta Mechanica Sinica, vol. 21, no. 5, pp. 444-450, 2005.

10. Габбасов Р.Ф. Численное построение разрывных решений задач строительной механики / Р.Ф. Габбасов, А.Р. Габбасов, В.В. Филатов. - М.: Изд-во АСВ, 2008. $230 \mathrm{c}$.

11. Варвак П.М., Варвак Л.П. Метод сеток в задачах расчета строительных конструкций. - М.: Стройиздат, 1977. 160 с. 


\title{
Aleksandrovskiy Maksim Vyacheslavovich \\ Moscow state university of civil engineering (national research university), Moscow, Russia \\ E-mail: AleksandrovskiyMV@mgsu.ru
}

\section{Use of generalized finite difference method for calculation of beams from nonlinear elastic material}

\begin{abstract}
The introduction into practice of construction of structures made of high-strength steels and other materials having a nonlinear deformation diagram caused the active development of the nonlinear theory of calculation of structures. Replacing Hooke's law with nonlinear dependencies between stresses and strains leads to so-called physical nonlinearity. For the calculation of such structures, the experimentally obtained dependences between stresses and strains are described using analytical expressions. A number of variants of such approximations have been proposed by various researchers. In this paper, we consider the calculation of beams of symmetrical cross-section made of nonlinear elastic material, for which the dependence between stresses and strains is described by a cubic parabola. This approximation ensures the symmetry of the diagram $\sigma$ with respect to the tension $\sigma-\varepsilon$ compression, and also gives a good match with the experimental curve. The use of the generalized finite difference method for solving the problem allows to reduce the system of nonlinear differential equations to the system of algebraic equations, for the solution of which the method of successive approximations is used. Studies have shown that the proposed method of calculation makes it possible to obtain a fairly accurate solution for a small number of elements. In addition, the presented calculation algorithm is convenient for programming and numerical calculation. As an example of systems that allow calculation using the considered algorithm, beam elements of building structures can act. The calculation of a beam from a nonlinear elastic material on the action of a concentrated force is given.
\end{abstract}

Keywords: calculation algorithm; nonlinear elastic material; cubic parabola approximation; generalized finite difference method; system of nonlinear differential equations; system of algebraic equations 


\section{REFERENCES}

1. Lukash P.A. (1978). Osnovy nelineynoy stroitel'noy mekhaniki. [Fundamentals of Nonlinear Structural Mechanics.] Moscow: Stroyizdat, p. 208.

2. Bondar' N.G. (1971). Nelineynye avtonomnye zadachi mekhaniki uprugikh sistem. [Nonlinear autonomous problems in the mechanics of elastic systems.] Kiev: Alarm Clock, p. 140.

3. Tsurpal I.A. (1976). Raschet ehlementov konstruktsiy iz nelineyno uprugikh materialov. [Calculation of structural elements from nonlinear elastic materials.] Kiev: "Technology", p. 176.

4. Crisfield M.A. (1994). Non-linear Finite Element Analysis of Solids and Structures. Wiley, (1), pp. 362.

5. Lee K. (2002). Large deflections of cantilever beams of non-linear elastic material under a combined loading. International Journal of Non-Linear Mechanics, 3(37), pp. 439-443.

6. Brojan M., Videnic T., Kosel F. (2009). Large deflections of nonlinearly elastic nonprismatic cantilever beams made from materials obeying the generalized Ludwick constitutive law. Meccanica, 6(44), pp. 733-739.

7. Solano-Carrillo E. (2009). Semi-exact solutions for large deflections of cantilever beams of non-linear elastic behaviour. International Journal of Non-Linear Mechanics, 2(44), pp. 253-256.

8. Petrov V.V., Krivosheyn I.V. (2009). Metody rascheta konstruktsiy iz nelineynodeformiruemogo materiala. [Methods for calculating structures from non-linearly deformable material.] Moscow: DIA Publishing House, p. 206.

9. Zhang X., Yang J. (2005). Inverse problem of elastica of a variable-arc-length beam subjected to a concentrated load. Acta Mechanica Sinica, 5(21), pp. 444-450.

10. Gabbasov R.F., Gabbasov A.R., Filatov V.V. (2008). Chislennoe postroenie razryvnykh resheniy zadach stroitel'noy mekhaniki. [Numerical construction of discontinuous solutions to problems of structural mechanics l.] Moscow: DIA Publishing House, p. 230.

11. Varvak P.M., Varvak L.P. (1977). Metod setok v zadachakh rascheta stroitel'nykh konstruktsiy. [The grid method in the problems of calculating building structures.] Moscow: Stroyizdat, p. 160. 